\title{
Functional Investigation of Solute Carrier Family 35, Member F2, in Three Cellular Models of the Primate Blood-Brain Barrier ${ }^{[\mathbb{s}}$
}

\author{
Tatsuki Mochizuki, ${ }^{2}$ ○Tadahaya Mizuno, ${ }^{2}$ Toshiki Kurosawa, Tomoko Yamaguchi, Kei Higuchi, ${ }^{1}$ \\ Yuma Tega, Yoshitane Nozaki, Kenji Kawabata, Yoshiharu Deguchi, and Hiroyuki Kusuhara
}

Laboratory of Molecular Pharmacokinetics, Graduate School of Pharmaceutical Sciences, The University of Tokyo, Tokyo, Japan (T.Mo., T.Mi., H.K.); Laboratory of Drug Disposition and Pharmacokinetics, Faculty of Pharma-Sciences, Teikyo University, Tokyo, Japan (T.K., K.H., Y.T., Y.D.); Laboratory of Stem Cell Regulation, National Institutes of Biomedical Innovation, Health and Nutrition, Osaka, Japan (T.Y., K.K.); and Drug Metabolism and Pharmacokinetics Tsukuba, Tsukuba Research Laboratories, Eisai Co., Ltd., Tsukuba, Ibaraki, Japan (Y.N.)

Received May 11, 2020; accepted October 8, 2020

\begin{abstract}
Understanding the mechanisms of drug transport across the bloodbrain barrier (BBB) is an important issue for regulating the pharmacokinetics of drugs in the central nervous system. In this study, we focused on solute carrier family 35, member F2 (SLC35F2), whose mRNA is highly expressed in the BBB. SLC35F2 protein was enriched in isolated mouse and monkey brain capillaries relative to brain homogenates and was localized exclusively on the apical membrane of MDCKII cells and brain microvascular endothelial cells (BMECs) differentiated from human induced pluripotent stem cells (hiPS-BMECs). SLC35F2 activity was assessed using its substrate, YM155, and pharmacological experiments revealed SLC35F2 inhibitors, such as famotidine (half-maximal inhibitory concentration, $160 \mu \mathrm{M})$. Uptake of YM155 was decreased by famotidine or SLC35F2 knockdown in immortalized human BMECs (human cerebral microvascular endothelial cell/D3 cells). Furthermore, famotidine significantly inhibited the apical (A)-to-basal (B) transport of YM155 in primary cultured monkey BMECs and hiPS-BMECs. Crucially, SLC35F2 knockout diminished the A-to-B transport and intracellular accumulation of YM155 in hiPS-BMECs. By contrast, in studies using
\end{abstract}

an in situ brain perfusion technique, neither deletion of S/c35f2 nor famotidine reduced brain uptake of YM155, even though YM155 is a substrate of mouse SLC35F2. YM155 uptake was decreased significantly by losartan and naringin, inhibitors for the organic anion transporting polypeptide (OATP) 1A4. These findings suggest SLC35F2 is a functional transporter in various cellular models of the primate $B B B$ that delivers its substrates to the brain and that its relative importance in the $B B B$ is modified by differences in the expression of OATPs between primates and rodents.

\section{SIGNIFICANCE STATEMENT}

This study demonstrated that SLC35F2 is a functional drug influx transporter in three different cellular models of the primate bloodbrain barrier (i.e., human cerebral microvascular endothelial cell/D3 cells, primary cultured monkey BMECs, and human induced pluripotent stem-BMECs) but has limited roles in mouse brain. SLC35F2 facilitates apical-to-basal transport across the tight cell monolayer. These findings will contribute to the development of improved strategies for targeting drugs to the central nervous system.

\section{Introduction}

It is well accepted that drugs must cross the blood-brain barrier (BBB), formed by a tight monolayer of brain microvascular endothelial

This study is supported by a Grant-in-Aid for Scientific Research (B) [Grant 26293032] and a Grant-in-Aid for Japan Society for the Promotion of Science Fellows [Grant 20J14855] from the Japan Society for the Promotion of Science.

${ }^{1}$ T.Mo. and T.Mi. contributed equally to this work.

${ }^{2}$ Current affiliation: Department of Cell Biology and Biochemistry, Texas Tech University Health Sciences Center, Lubbock, Texas.

The authors declare no conflict of interest.

https://doi.org/10.1124/dmd.120.000115.

S This article has supplemental material available at dmd.aspetjournals.org. cells (BMECs), from the blood circulation to reach their targets in the central nervous system (CNS). Hence, understanding the mechanisms associated with drug transport across the $\mathrm{BBB}$ is critically important for the development of effective CNS drugs. Efflux transporters such as $\mathrm{P}$-glycoprotein (P-gp) and breast cancer resistance protein (BCRP) have been well characterized in both preclinical animal and human BBB models and reduce the distribution of their substrate drugs within the CNS via their transport activities (Demeule et al., 2002; Sasongko et al., 2005; Nicolazzo and Katneni, 2009). Moreover, many researchers have investigated transporters that can facilitate the transfer of drugs from the blood to the CNS. For instance, the function of organic anion transporting polypeptide (OATP) 1A4 has been extensively investigated preclinically. However, in our previous report, convincing data were not

ABBREVIATIONS: BBB, blood-brain barrier; BCRP, breast cancer resistance protein; BMEC, brain microvascular endothelial cell; CNS, central nervous system; HA, human influenza hemagglutinin; hCMEC, human cerebral microvascular endothelial cell; HEK, human embryonic kidney; hiPS, human induced pluripotent stem; $\mathrm{H}^{+}$/OC, proton/organic cation; hSLC35F2, human solute carrier family 35, member F2; MDCK, Madin-Darby canine kidney; mSLC35F2, mouse solute carrier family 35, member F2; OATP, organic anion transporting polypeptide; OCT, organic cation transporter; $P_{\text {app }}$, apparent permeability coefficient; $P_{\text {dif, }}$ nonsaturable uptake clearance; P-gp, P-glycoprotein; siRNA, small interfering RNA; SLC,

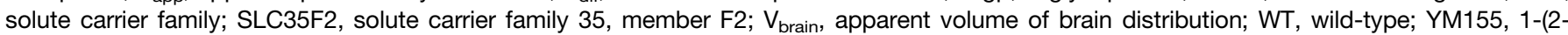
Methoxyethyl)-2-methyl-4,9-dioxo-3-(pyrazin-2-ylmethyl)- 4,9-dihydro-1H-naphtho[2,3- $d$ ]imidazolium. 
A

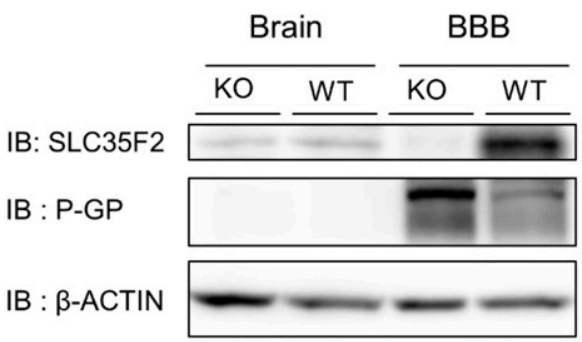

B

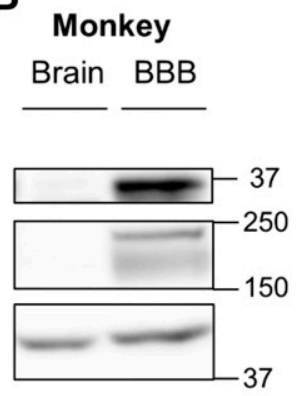

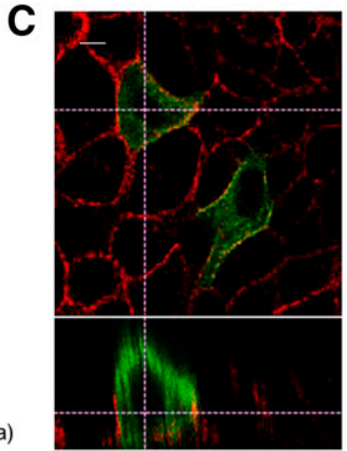

Fig. 1. SLC35F2 expression in the BBB and intracellular localization in MDCKII cells. (A and $\mathrm{B})$ Immunoblotting to confirm protein expression of mouse (A) and monkey (B) SLC35F2 in brain homogenate (Brain) and crude membrane fractions of brain capillaries (BBB) inSlc35f2 ${ }^{--}$(KO) and wild-type (WT) mouse specimens. P-gp was detected as a marker of the crude membrane fractions of BMECs. (C) hSLC35F2-HA localization in MDCKII cells detected by confocal laser scanning microscopy. Green and red fluorescence represent HA tag and $\mathrm{Na}^{+} / \mathrm{K}^{+}$ATPase (basolateral membrane marker), respectively. The upper section illustrates the horizontal image, and the lower section represents the vertical image. Scale bars represent $10 \mu \mathrm{m}$. The experiments were repeated at least twice. IB, immunoblotting; $\mathrm{KO}$, knockout available to support its targeting efficacy for organic anions in vivo (Ose et al., 2010). On the other hand, the distribution of [D-penicillamine ${ }^{2,5}$ ] enkephalin, a substrate of OATP1A4 within the brain, was consistently altered by changes in OATP1A4 expression under normal conditions and those mimicking disease. In addition, the distribution was decreased by OATP inhibitors, suggesting a role for OATP1A4 in targeting drugs to the brain (Ronaldson et al., 2011)(Ronaldson et al., 2011). It has also been speculated that several hydrophobic, cationic drugs, such as pyrilamine, oxycodone, and tramadol, can cross the BBB via a proton/organic cation antiporter(s) (Okura et al., 2008; Kitamura et al., 2014; Higuchi et al., 2015). In another report, it was demonstrated that some D2 dopamine receptor antagonists, i.e., olanzapine, haloperidol, and risperidone, displayed higher receptor occupancy in the cortex than in the human pituitary (Arakawa et al., 2010). This finding suggested unknown mechanism(s) in the brain that cause higher unbound drug concentrations in the cerebral cortex than in the pituitary. These include active transporters in the BBB to deliver drugs into the CNS because these drugs can freely access their targets from the blood in the pituitary.

In the present study, we focused on solute carrier family 35, member F2 (SLC35F2), which is highly expressed in BMECs compared with endothelial cells in other tissues and other brain cells in mice (Daneman et al., 2010; Zhang et al., 2014). SLC35F2 was initially identified as a membrane protein acting as a retroviral receptor (Sarangi et al., 2007). Subsequently, SLC35F2 was identified by genome-wide screening in KBM7 cells as a transporter for a Survivin inhibitor, YM155, which sensitizes KBM7 as well as various other cells to YM155 cytotoxicity (Winter et al., 2014). With regard to other SLC-type transporters accepting YM155 as a substrate, organic cation transporter (OCT) 1 and OCT2 have been identified as candidates to account for the uptake of YM155 in xenobiotic clearance organs, such as liver and kidney, respectively (Minematsu et al., 2010). In addition, YM155 is also recognized as a substrate by P-gp (Iwai et al., 2011), which conferred resistance to YM155 (Radic-Sarikas et al., 2017).

In the present study, we aimed to characterize SLC35F2 as a drug transporter in the BBB. To characterize SLC35F2 transport function, we constructed HEK293 cells stably expressing SLC35F2 and SLC35F2 knockout PC-3 cells (Winter et al., 2014). We assessed SLC35F2 activity in three cellular models of the BBB: 1) hCMEC/D3 cells, immortalized human BMECs (Weksler et al., 2013); 2) a cellular model of monkey BBB composed of primary cultured monkey BMECs and rat pericytes and astrocytes; and 3) BMECs derived from human induced pluripotent stem cells (hiPS-BMECs) (Lippmann et al., 2012, 2014; Kurosawa et al., 2018). In addition, the role of SLC35F2 in vivo was investigated using $\operatorname{Slc} 35 f 2^{-/-}$mice.

\section{Materials and Methods}

Reagents. YM155 monobromide was purchased from Funakoshi Co. (Tokyo). All other chemicals and reagents were of reagent grade and commercially available.

Cell Culture. HEK293 and MDCKII cells were cultured in Dulbecco's modified Eagle's medium (low glucose; Invitrogen, Carlsbad, CA) supplemented with $10 \%$ fetal bovine serum. PC-3 cells (CRL-1435; American Type Culture Collection) were cultured in RPMI 1640 medium (Invitrogen) supplemented with $10 \%$ fetal bovine serum. hCMEC/D3 cells were maintained on collagen-coated dishes as reported previously (Okura et al., 2014). MBT-24H (lot: 24E50), which we abbreviated as monkey BMECs, was purchased from Pharmaco-Cell Co. (Nagasaki, Japan). This model was composed of monkey BMECs and rat pericytes and astrocytes and was maintained according to the manufacturer's protocol (Nakagawa et al., 2007). Transcellular transport analysis using monkey BMECs was conducted on day 5 of the culture. The hiPS cell line (IMR90-C4; WiCell, Madison, WI) was cultured in mTeSR1 medium without human fibroblast growth factor 2 in a Matrigel-coated dish. The differentiation to hiPS-BMECs was conducted as described previously (Lippmann et al., 2012, 2014; Kurosawa et al., 2018). All cells were maintained at $37^{\circ} \mathrm{C}$ under an atmosphere of $5 \% \mathrm{CO}_{2}$ in air with $95 \%$ humidity.

TABLE 1

Kinetic parameters of YM155

The kinetic parameters of YM155 uptake in hSLC35F2-HEK293 and mSLC35F2-HEK293 cells (Supplemental Fig. 1D) and parental hCMEC/D3 cells (Fig. 2B) The values represent mean \pm computer-calculated S.D. The experiments were repeated at least twice.

\begin{tabular}{lccc}
\hline & $K_{\mathrm{m}, \text { app }}$ & $V_{\max , \text { app }}$ & $P_{\text {dif }}$ \\
\hline$\mu M$ & pmol/min per milligram protein & $\mu l /$ min per milligram protein \\
$\mathrm{hSLC35F2}$ & $0.310 \pm 0.038$ & $15.2 \pm 1.1$ & $2.41 \pm 0.21$ \\
$\mathrm{mSLC35F2}$ & $1.55 \pm 0.36$ & $97.1 \pm 20.9$ & $2.47 \pm 1.68$ \\
$\mathrm{hCMEC/D3}$ & $0.573 \pm 0.166$ & $1.74 \pm 0.35$ & $0.277 \pm 0.046$ \\
\hline
\end{tabular}


Animals. Slc $35 \mathrm{f2}^{-/-}$mice were established using the CRISPR/Cas9 gene knockout system in the Laboratory Animal Resource Center, University of Tsukuba (Ibaraki, Japan). Male C57BL/6J were purchased from CLEA Japan (Tokyo). All mice were maintained at a controlled temperature under a 12-hour light/ dark cycle. Food and water were available ad libitum. All experiments using animals in this study were performed according to the guidelines provided by the Institutional Animal Care Committee (Graduate School of Pharmaceutical Sciences, University of Tokyo, Tokyo, Japan). Monkey tissues were prepared from young, adult (approximately 10 years old), non-naïve male cynomolgus macaques (Macaca fascicularis) by the procedures approved by the Institutional Animal Care and Use Committee of Eisai Tsukuba Research Laboratories (Ibaraki, Japan).

Construction of SLC35F2 Knockout PC-3 Cells. The gene knockout in the PC-3 cells was produced using the CRISPR/Cas9 system. The CRISPR oligo sequences used are listed in Supplemental Table 1. The cell-construction method used was similar to that reported previously (Winter et al., 2014), with some modifications, and the details are shown in Supplemental Methods.

Isolation of Crude Membrane-Enriched Fraction of Isolated Brain Capillaries. The protocol used to isolate crude membrane fractions of brain capillaries was followed as described previously (Ose et al., 2010). All steps were conducted at $4^{\circ} \mathrm{C}$. Monkey specimens were derived from a single brain hemisphere and mouse samples were from the whole brains of eight male mice (9 to 10 weeks old).

Immunoblotting Analysis. Specimens were lysed using Cell Lysis Buffer (Cell Signaling Technology, Danvers, MA) and reduced for 5 minutes at $60^{\circ} \mathrm{C}$ with $40 \mu \mathrm{l}$ of SDS sample buffer (New England BioLabs, Beverly, MA) containing 2mercaptoethanol. Samples were loaded into wells of a $10 \%$ SDS-polyacrylamide gel with a 3.75\% stacking gel, electrophoresed, and subjected to Western blot analysis, as described previously (Mizuno et al., 2015). SLC35F2 was detected using a 1:1000 dilution of primary antibody (HPA048185; Merck, Darmstadt, Germany).

Immunocytochemistry. HEK293 cells and PC-3 cells were seeded on glass coverslips (Matsunami Glass Industries, Osaka, Japan) in 12-well plates at a density of $1.0 \times 10^{5}$ cells per well for 24 hours. Similarly, MDCKII cells were seeded at a density of $5.0 \times 10^{5}$ cells per well and cultured for 48 hours to construct a monolayer structure. MDCKII cells were transfected with pcDNA3.1(+) vector containing hSLC35F2-HA or mSLC35F2-HA at 24 hours after seeding. hiPS-BMECs were prepared using the same protocol as the transcellular transport assay. Immunocytochemical assays were conducted as described previously (Hayashi et al., 2016). Cells were fixed in 4\% paraformaldehyde/PBS for 10 minutes, permeabilized in $0.1 \%$ saponin/PBS for 10 minutes, and blocked with $3 \%$ bovine serum albumin/PBS for 30 minutes, followed by 2 hours of incubation at room temperature with primary antibodies: anti-HA (1: 250, 3F10; Roche Diagnostics, Mannheim, Germany), anti-SLC35F2 (1:20, HPA048185; Merck), or anti- $\mathrm{Na}^{+} / \mathrm{K}^{+}$ATPase (1:20, C464.6; Merck). After washing with PBS, cells were incubated with Alexa Fluor 488/546/555conjugated secondary antibody and TO-PRO-3 (Thermo Fisher Scientific,

TABLE 2

Effects of organic cations on YM155 uptake in PC-3 and hCMEC/D3 cells

We determined 5 min YM155 $(0.1 \mu \mathrm{M})$ uptake mediated by SLC35F2 in control PC-3 or hCMEC/D3 cells in the absence and presence of the test compounds. Values represent mean \pm S.E. $(n=3$ to 4$)$. N.D. indicates no data. The experiments were repeated at least twice.

\begin{tabular}{lccc}
\hline \multirow{2}{*}{ Conditions/Compounds } & \multirow{2}{*}{ Conc. } & \multicolumn{2}{c}{ Control YM155 Uptake (\%) } \\
\cline { 3 - 4 } & & PC-3 & hCMEC/D3 \\
\hline Control & Free & $100 \pm 6$ & $100 \pm 8$ \\
SLC35F2 knockout & & $10.3 \pm 1.0^{* * *}$ & N.D. \\
L-carnitine & $1 \mathrm{mM}$ & $125 \pm 14^{* *}$ & $120 \pm 4^{*}$ \\
TEA & & $83.4 \pm 1.8$ & $64.2 \pm 4.9^{* *}$ \\
Varenicline & & $76.4 \pm 3.0^{* *}$ & $84.3 \pm 10.4$ \\
Famotidine & & $16.4 \pm 1.8^{* *}$ & $15.1 \pm 0.6^{* *}$ \\
Tramadol & $13.0 \pm 1.0^{* *}$ & $32.6 \pm 5.6^{* *}$ \\
Clonidine & & $9.06 \pm 0.47^{* *}$ & $21.9 \pm 1.3^{* *}$ \\
Diphenhydramine & & $5.65 \pm 0.32^{* *}$ & $12.4 \pm 0.7^{* *}$ \\
Pyrilamine & & $5.46 \pm 0.97^{* *}$ & $14.1 \pm 1.3^{* *}$ \\
Imipramine & & $5.36 \pm 0.34^{* *}$ & $34.4 \pm 2.5^{* *}$ \\
Verapamil & $100 \mu \mathrm{M}$ & $5.11 \pm 0.28^{* *}$ & $27.0 \pm 6.1^{* *}$ \\
Quinidine & & $3.22 \pm 0.51^{* *}$ & $15.1 \pm 0.6^{* *}$ \\
\hline
\end{tabular}

${ }^{*} P<0.05 ;{ }^{* *} P<0.01$ vs. control.
Waltham, MA) for 1 hour at room temperature. Cells were mounted onto glass slides with Vectashield mounting medium (Vector Laboratories, Burlingame, CA) and then visualized by confocal microscopy using a Leica TCS SP5 II laser scanning confocal microscope (Leica, Solms, Germany).

In Vitro Uptake Assay. In vitro uptake studies with HEK293, PC-3, and hCMEC/D3 cells were conducted as reported previously (Deguchi et al., 2004; Tsuruya et al., 2016). To determine the uptake of compounds, the cells were recovered in water using a scraper and disrupted by sonication. A 3-fold volume of acetonitrile was added for deproteinization, and the specimens were centrifuged for 5 minutes at $20,000 \mathrm{~g}$. The protein concentration was determined using the Lowry method with bovine serum albumin as the standard.

In Vitro Permeability Test. In vitro permeability tests using monkey BMECs and hiPS-BMECs were conducted as described previously (Kurosawa et al., 2018). The apparent permeability coefficient $\left(P_{\mathrm{app}}\right)$ was calculated as follows:

$$
P_{\text {app }}=\frac{\mathrm{d} Q / \mathrm{d} t}{D_{0} \times S}
$$

where $\mathrm{d} Q / \mathrm{d} t, D_{0}$, and $S$ are the slope of the linear region of the cumulative amount of permeant in the receiver chamber versus time plot, the starting concentration of the drug on the donor side, and the area of the porous membrane, respectively. Compounds accumulated in the cell were collected by treatment with methanol for 10 minutes at room temperature.

In Situ Brain Perfusion Test in Mice. In situ brain perfusion in mice was performed as described previously (Dagenais et al., 2000; Ose et al., 2010; Sano et al., 2018), and the details are available in the Supplemental Methods.

Quantification of Test Compounds. Lucifer yellow was detected using an Infinite M200 spectrometer (428/536 nm; Tecan, Crailsheim, Germany) or liquid chromatography with a fluorescence detector composed of a Nexera system equipped with an RF-20Axs (Shimadzu, Kyoto, Japan). Other compounds were quantified using liquid chromatography-tandem mass spectrometry. A SCIEX QTRAP 5500 mass spectrometer (Applied Biosystems, Foster City, CA) equipped with a Prominence LC system (Shimadzu) and operated in electrospray ionization mode was used for the analysis. The conditions are summarized in Supplemental Table 2.

Kinetic Analysis of Data Obtained In Vitro. The uptake in the transporterexpressing cells was subjected to the kinetic analyses without subtraction of the uptake in the mock cells. The curve fitting was conducted with the iterative nonlinear least-squares method using the MULTI program (Yamaoka et al., 1981) and the damped Gauss-Newton algorithm.

The apparent kinetic parameters were estimated assuming the MichaelisMenten equation as follows:

$$
v=\frac{V_{\text {max,app }} \times \mathrm{S}}{K_{\mathrm{m}, \text { app }}+\mathrm{S}}+P_{\mathrm{dif}} \times \mathrm{S},
$$

where $v, K_{\mathrm{m} \text {, app }}, S, V_{\mathrm{max}, \text { app }}$, and $P_{\mathrm{dif}}$ represent the uptake velocity, Michaelis constant, substrate concentration, maximum transport velocity, and nonsaturable uptake clearance, respectively. The $\mathrm{IC}_{50}$ values against YM155 uptake for hSLC35F2 were calculated as follows:

$$
C L_{\text {uptake(+Inhibitor) }}=\frac{C L_{\text {uptake(control) }}}{1+I / \mathrm{IC}_{50}},
$$

where $C L_{\text {uptake (+Inhibitor) }}$ and $C L_{\text {uptake (control) }}$ represent the uptake clearance in the presence and absence of inhibitors, respectively, and $I$ represents the inhibitor concentration.

Data Analysis. The statistical significance of differences was determined using a Welch $t$ test and one-way analysis of variance followed by a Tukey or Dunnett multiple-comparison test. Data were analyzed using Prism software (version 4.0; GraphPad Software, La Jolla, CA).

\section{Results}

Expression of SLC35F2 in the BBB and Localization in MDCKII Cells. Immunoblotting analysis using anti-SLC35F2 antibody revealed that a band around $37 \mathrm{kDa}$ was concentrated in the crude membrane-enriched fraction of isolated brain capillaries compared with 
TABLE 3

Half-maximal inhibitory concentration against YM155 uptake mediated by SLC35F2

Values represent $\mathrm{IC}_{50}$ of the compounds against 5 min YM155 $(0.1 \mu \mathrm{M})$ uptake mediated by SLC35F2 in control PC-3 cells. Detailed data are presented in Supplemental Fig. 3. Values represent mean \pm computer-calculated S.D. The experiments were repeated at least twice.

\begin{tabular}{lc}
\hline Compound & $\mathrm{IC}_{50}$ \\
\hline & $\mu M$ \\
Famotidine & $160 \pm 28$ \\
Pyrilamine & $101 \pm 27$ \\
Clonidine & $77.7 \pm 14.6$ \\
Diphenhydramine & $65.7 \pm 6.8$ \\
Imipramine & $17.2 \pm 5.2$ \\
Quinidine & $4.01 \pm 0.73$ \\
\hline
\end{tabular}

the corresponding fraction of brain homogenates from wild-type (WT) mice, and a similar concentration was not observed in specimens from Slc35f $2^{-1-}$ mice (Fig. 1A). In addition, the same size band was detected in the crude membrane-enriched fraction of isolated brain capillaries from monkey brain (Fig. 1B).

We conducted an immunohistochemical study of SLC35F2 in a mouse brain, but specific signals associated with SLC35F2 were not detected (data not shown). In C-terminal HA-tagged hSLC35F2 (hSLC35F2HA)-transfected MDCKII cells, which are polarized when constructing a tight cell monolayer, hSLC35F2-HA was detected mainly on the apical membrane of the cells (Fig. 1C), and mSLC35F2-HA produced a similar result (data not shown). These data suggest the localization of SLC35F2 on the luminal membrane (corresponding to the apical membrane) in endothelial cells.

YM155 Transport Function Mediated by hSLC35F2 and mSLC35F2. We established HEK293 cell clones stably expressing hSLC35F2-HA (hSLC35F2-HEK293) or mSLC35F2-HA (mSLC35F2HEK293), as described in the Supplemental Methods. SLC35F2 expression was confirmed by immunoblotting (Supplemental Fig. 1A) and immunocytochemistry (Supplemental Fig. 1B). YM155 uptake was significantly increased in both cell lines compared with mocktransfected HEK293 cells (Supplemental Fig. 1C). Specific YM155 uptake mediated by SLC35F2 was determined at 5 minutes in a subsequent analysis, as the initial uptake velocities were maintained during this time. The concentration dependence of YM155 uptake is illustrated as Eadie-Hofstee plots (Supplemental Fig. 1D), and the calculated kinetic parameters displayed little species difference (Table 1). The uptake curve extrapolated to time zero did not pass through the origin, suggesting that our analysis of uptake velocity may have underestimated the initial uptake velocity. Thus, the kinetic parameters represent apparent values.

Effect of Organic Cations on YM155 Uptake Facilitated by SLC35F2 in PC-3 Cells. YM155 uptake was detected even in mocktransfected HEK293 cells (about $60 \mu \mathrm{l} / 5 \mathrm{~min}$ per milligram protein), accounting for $27 \%$ of the uptake in hSLC35F2-HEK293 (Supplemental Fig. 1C). To evaluate the specific hSLC35F2 activity more precisely, we constructed SLC35F2 knockout cells using PC-3 cells that express high endogenous levels of hSLC35F2 (Winter et al., 2014). Depletion of SLC35F2 was confirmed by immunoblotting and immunocytochemistry analysis (Supplemental Fig. 2, A and B), and the SLC35F2 deficiency dramatically decreased YM155 uptake activity to $10 \%$ of control cells (Table 2).

In studies to characterize SLC35F2-mediated transport, the effects of various organic cations on YM155 uptake were examined in PC-3 cells (Table 2). Some of the selected compounds were reported to inhibit the function of organic cation transporters. For example, L-carnitine has been shown to inhibit the organic cation/carnitine transporters, and TEA inhibits OCTs (Koepsell et al., 2007). In contrast to their effect on these transporters, L-carnitine and TEA had no effect or only a small effect on YM155 uptake mediated by SLC35F2. By contrast, other drugs did inhibit SLC35F2-mediated transport, and the results for some compounds were consistent with a previous report (Minematsu et al., 2009). Apart from famotidine, all the effective drugs were shown to inhibit the unknown proton/organic cation $\left(\mathrm{H}^{+} / \mathrm{OC}\right)$ antiporter(s), reported to be functional in the BBB (Okura et al., 2008; Kitamura et al., 2014; Suzuki et al., 2016; Kurosawa et al., 2017). To determine their relative inhibitory potency, we calculated half-maximal inhibitory concentration values for famotidine, clonidine, diphenhydramine, pyrilamine, imipramine, and quinidine (Supplemental Fig. 3; Table 3).

Investigation of SLC35F2 Substrates Other than YM155. To examine whether SLC35F2 transports various xenobiotics, we conducted uptake studies in two cellular models: mock-transfected HEK293 cells versus mSLC35F2-HEK293 cells, and control versus SLC35F2 knockout PC-3 cells. Among the tested compounds, only the uptake of famotidine was significantly altered in both cellular models (Supplemental Fig. 4A). Famotidine uptake was significantly inhibited by excess YM155 in mSLC35F2-HEK293 cells (Supplemental Fig. 4B), consistent with SLC35F2-mediated uptake.

Compounds that suppressed YM155 uptake in PC-3 cells included many hydrophobic cationic drugs (Table 2), which are easily accumulated intracellularly and thereby possibly affect the intracellular YM155 distribution to acidic subcellular organelles such as lysosomes (Kubo et al., 2016). Among the SLC35F2 inhibitors studied, famotidine was identified as a novel substrate of SLC35F2 (Supplemental Fig. 4B), which acts as a competitive inhibitor. Therefore, we used famotidine as an inhibitor for SLC35F2 in subsequent analyses.

Characterization of YM155 Uptake in hCMEC/D3 Cells. To investigate whether the activity of SLC35F2 is maintained in the BBB, we conducted a functional analysis with hCMEC/D3 cells, which are an immortalized cell line of human BMECs (Weksler et al., 2005; Shimomura et al., 2013). To characterize YM155 uptake in hCMEC/ D3 cells, the time course and concentration dependence of YM155 uptake were investigated in hCMEC/D3 cells (Fig. 2, A and B; Table 1). The $K_{\mathrm{m}}$, app in hCMEC/D3 cells $(0.57 \mu \mathrm{M})$ was close to that of hSLC35F2 $(0.31 \mu \mathrm{M}$, Table 1$)$, whereas the affinity for other YM155 transporters was $22.1 \mu \mathrm{M}$ for OCT1 and $2.67 \mu \mathrm{M}$ for OCT2 (Minematsu et al., 2010). The profile of inhibitory effects on YM155 uptake in hCMEC/D3 cells was similar to that in PC-3 cells (Table 2). We also examined the effect on YM155 uptake of SLC35F2 knockdown by siRNA treatment. SLC35F2 protein expression was diminished in the whole lysate specimen of SLC35F2 siRNAtreated cells (Fig. 2C). The fraction of YM155 uptake inhibited by famotidine (i.e., the difference in uptake between famotidine treated and nontreated conditions) was decreased by $64 \%$ by SLC35F2 knockdown, supporting the significance of SLC35F2 in YM155 uptake in hCMEC/D3 cells (Fig. 2D).

The A-to-B Transcellular Transport of YM155 Across Monkey BMECs. hCMEC/D3 cells do not form a tight monolayer, which limits their application to study the directional transport of YM155 mediated by SLC35F2 (Srinivasan et al., 2015). Therefore, we employed a commercial cellular model of the primate BBB, i.e., monkey BMECs (MBT-24H). SLC35F2 mRNA expression in monkey BMECs was confirmed by polymerase chain reaction analysis (Fig. 3A). The transendothelial electrical resistance was above $330 \Omega \times \mathrm{cm}^{2}$ in all samples. In addition, we measured the amounts of transported Lucifer yellow as a paracellular transport marker (Supplemental Fig. 5A). In the presence of $10 \mu \mathrm{M}$ Lucifer yellow, transport levels (25-35 pmol/4 hours) were low compared with those ( $\sim 480 \mathrm{pmol} / 4$ hours $)$ in other cellular models of the BBB, such as hCMEC/D3 and bEnd3 cells 


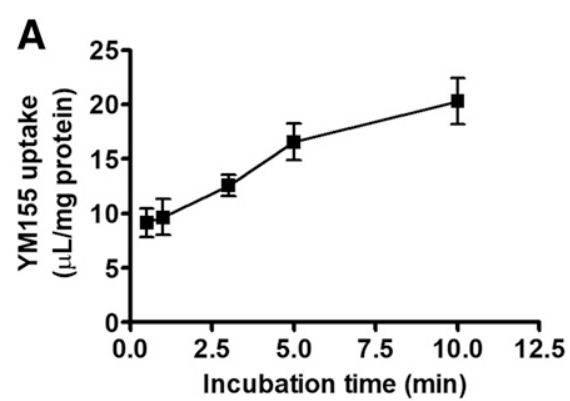

C

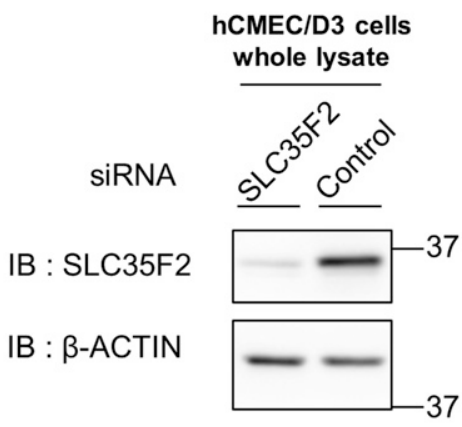

B

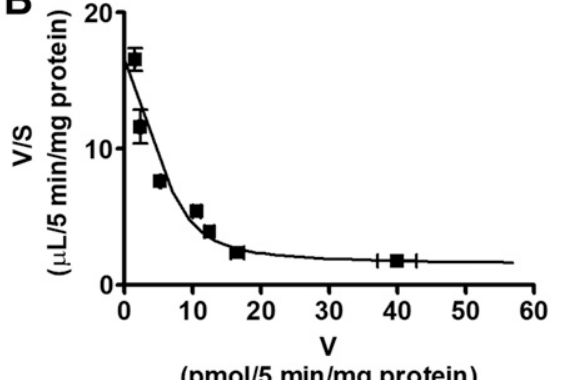

D

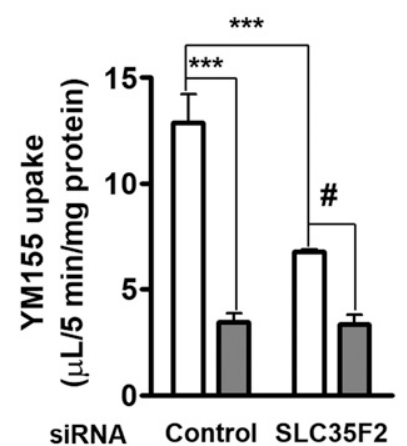

Fig. 2. YM155 uptake mediated by hSLC35F2 in hCMEC/D3 cells. (A) Time course of YM155 $(0.1 \mu \mathrm{M})$ uptake in parent hCMEC/D3 cells. (B) Eadie-Hofstee plots for 5 minutes of YM155 uptake in hCMEC/D3 cells. The line represents fitted curves. Details described in Materials and Methods. The plots were accounted for by a saturable and nonsaturable component. The fitted $K_{\mathrm{m} \text {, app }}, V_{\mathrm{max} \text {, app }}$, and $P_{\mathrm{dif}}$ calculated were shown in Table 1. (C and D) Effects of SLC35F2 siRNA on YM155 uptake in hCMEC/D3 cells. Cells were treated with $10 \mathrm{nM}$ negative control or SLC35F2 siRNA using LipofectAMINE RNAiMAX for 48 hours. (C) Immunoblotting of hCMEC/D3 whole cell lysate to investigate endogenous SLC35F2 expression and $10 \mathrm{nM}$ SLC35F2 siRNA efficiency. (D) The uptake of YM155 $(0.1 \mu \mathrm{M})$ was measured for 5 minutes. Open bars illustrate the results under no inhibitor-treated conditions, and the gray bars indicate the results under $1 \mathrm{mM}$ famotidine-treated conditions. The error bars represent the S.E.M. $(n=4$, mean \pm S.E. $)$. $* * * P<$ 0.001 vs. control without inhibitor. ${ }^{\#} P<0.05$ vs. SLC35F2 knockdown without inhibitor. The experiments were repeated at least twice. IB, immunoblotting.
(Eigenmann et al., 2013; Yang et al., 2018). Therefore, we concluded that monkey BMECs can form a tight junction.

Drug transporter function in monkey BMECs was also confirmed by directional transport of quinidine, a typical reference substrate of P-gp, the B-to-A transport of which was greater than the A-to-B transport because of active efflux by P-gp in the apical membrane (Kurosawa et al., 2018) (Supplemental Fig. 5B). Likewise, the amount of YM155 B-to-A transport was about 3-fold higher than its A-to-B transport (data not shown). In regard to YM155 A-to-B transport, the amount was significantly decreased by $1 \mathrm{mM}$ famotidine (Fig. 3B). In addition, the intracellular accumulation of YM155 was suppressed by famotidine from 0.03 pmol per well to an undetectable level ( $\leq 0.02$ pmol per well) (Fig. 3C).

Characterization of YM155 Transcellular Transport in hiPS-BMECs. Recently, hiPS-BMECs were established as an emerging cellular model of the human BBB (Lippmann et al., 2012, 2014). Subsequently, we demonstrated that hiPS-BMECs express various transporters and demonstrated directional transport of nutrients and prazosin across a tight cell monolayer (Kurosawa et al., 2018). In the current study, we found that SLC35F2 protein expression was induced during the differentiation process and achieved a higher level than that observed in hCMEC/D3 cells (Fig. 4A). Lucifer yellow transport in hiPSBMECs was small, even compared with that in monkey BMECs, indicating that paracellular transport was strictly regulated in hiPSBMECs (Supplemental Fig. 5A; Supplemental Fig. 6A). As a control for transcellular transport, B-to-A directional transport of dantrolene, a typical BCRP substrate (Enokizono et al., 2008), was confirmed as reported (Kurosawa et al., 2018) (Supplemental Fig. 6B).

A clear A-to-B directional transport of YM155 was observed, which was markedly suppressed by $1 \mathrm{mM}$ famotidine (Fig. 4B). Along with inhibition of the directional transport (Fig. 4C), famotidine significantly suppressed YM155 intracellular accumulation (Fig. 4D), whereas other test compounds, including TEA for OCTs, L-carnitine for organic cation/ carnitine transporters, thiamine for OCTs and thiamine transporters
A

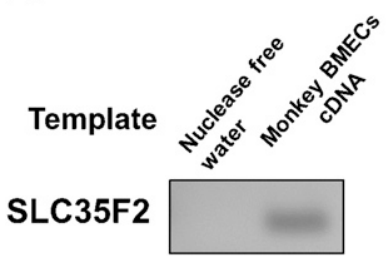

B

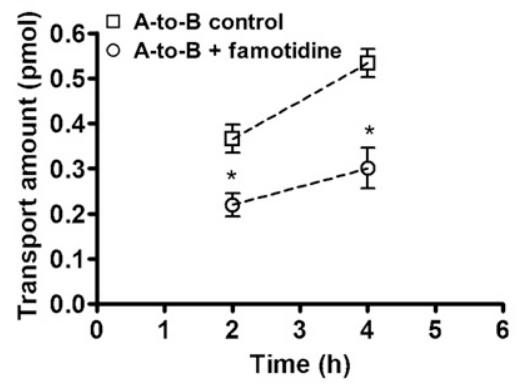

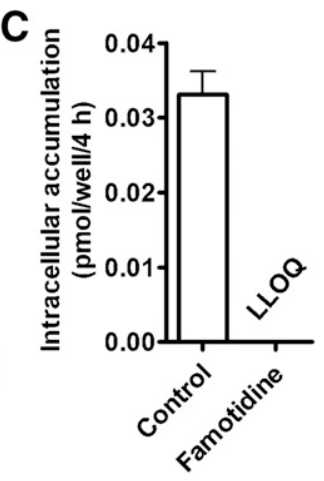

Fig. 3. YM155 permeability across monkey BMECs (MBT-24H). (A) SLC35F2 mRNA expression in monkey BMECs. Conventional polymerase chain reaction analysis was conducted with nuclease-free water and cDNA derived from isolated monkey brain microvascular endothelial cells constituting MBT-24H. (B) YM155 $(0.1 \mu \mathrm{M})$ permeable amount at 2 and 4 hours across monkey BMECs. The open squares and open circles represent A-to-B permeable amount of control and $1 \mathrm{mM}$ famotidine-treated cells, respectively. (C) YM155 $(0.1 \mu \mathrm{M})$ intracellular accumulation after 4 hours permeability test. LLOQ indicates levels lower than the limit of quantification $(0.02 \mathrm{pmol} / \mathrm{well}$ per 4 hours). The error bars represent the S.E.M. $(n=3$, mean \pm S.E.). $* P<0.05$ vs. permeable amount of control A-to-B direction. The experiments were conducted once using lot 24E50. 

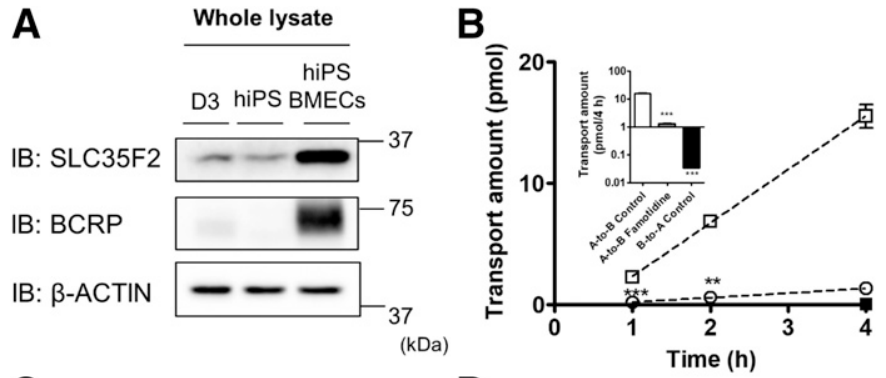

C
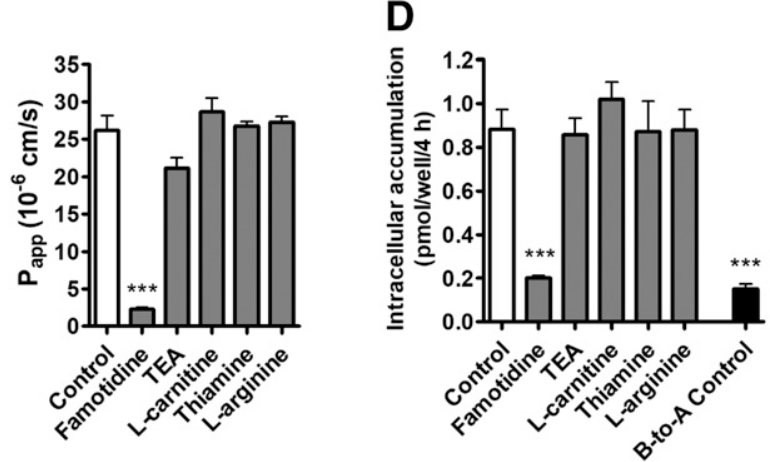

Fig. 4. YM155 permeability across hiPS-BMECs and the effects of cationic compounds. (A) Immunoblotting of whole cell lysates of hiPS-BMECs confirmed their SLC35F2 protein expression compared with hCMEC/D3 cells (D3) and undifferentiated hiPS cells (hiPS). (B) YM155 (0.1 $\mu \mathrm{M})$ permeable amount across hiPS-BMECs. The open and closed squares represent the transport amount of A-to$\mathrm{B}$ and B-to-A directions, respectively, and open circles represent A-to-B permeable amount with the $1 \mathrm{mM}$ famotidine treatment. The bars represent YM155 permeable amount at 4 hours. (C and D) Calculated permeability coefficient (C) and intracellular accumulation (D) of YM155 with the treatment of various organic cations, $1 \mathrm{mM}$ famotidine, $500 \mu \mathrm{M}$ TEA, $500 \mu \mathrm{M}$ L-carnitine, $500 \mu \mathrm{M}$ thiamine, and $1 \mathrm{mM} \mathrm{L}$-arginine. Open and gray bars represent the data for the A-to-B direction, and the black bar represents the data for the B-to-A direction. The error bars represent the S.E.M. $(n=3$, mean \pm S.E. $)$. $* * P<0.01$; $* * * P<0.001$ vs. control value of A-to-B direction. The experiments were repeated at least twice. IB, immunoblotting.

(SLC19A2, SLC19A3), and L-arginine for cationic amino acid transporter 1 (SLC7A1), did not affect the A-to-B transport or intracellular accumulation of YM155 (Fig. 4, C and D).

YM155 Transcellular Transport in SLC35F2 Knockout hiPS-BMECs. To elucidate the role of SLC35F2 directly in hiPSBMECs, we constructed a SLC35F2 knockout IMR90-C4 cell clone and conducted several assays after differentiation to SLC35F2 knockout hiPS-BMECs. Firstly, the absence of SLC35F2 protein was confirmed in SLC35F2 knockout hiPS-BMECs (Fig. 5A). Using this cell as a negative control, specific SLC35F2 signal was confirmed on the apical membrane of hiPS-BMECs (Fig. 5, B and C). The low permeability of Lucifer yellow was maintained in SLC35F2 knockout hiPS-BMECs, although the absolute values were slightly increased compared with those in control cells (Supplemental Fig. 7A). Thus, SLC35F2 knockout apparently does not affect BCRP protein expression and transport activity in hiPS-BMECs (Fig. 5A; Supplemental Fig. 7B).

The A-to-B permeability of YM155 was dramatically decreased in SLC35F2 knockout hiPS-BMECs (Fig. 5D). The permeability coefficient and the intracellular accumulation in SLC35F2 knockout hiPSBMECs were decreased to approximately $2 \%$ and $3 \%$ of the corresponding values in the control cells, respectively (Fig. 5, E and F), indicating the predominant contribution of SLC35F2 to the uptake of YM155 from the apical side. Notably, these results were reproduced in another clone of SLC35F2 knockout hiPS-BMECs (data not shown).

Characterization of YM155 Transport Across the Mouse BBB. Subsequently, we examined the function of SLC35F2 in vivo. Because
YM155 transport was confirmed for mSLC35F2 (Table 1), we conducted an in situ brain perfusion test to characterize the BBB transport of YM155 in mice. In WT mice, the apparent volume of brain distribution $\left(\mathrm{V}_{\text {brain }}\right)$ of YM155 was decreased according to the concentration in the perfusate (Fig. 6A), which suggests that uptake into the brain from the perfusate or the tissue binding of YM155 was saturated. However, the $\mathrm{V}_{\text {brain }}$ of YM155 was unchanged by Slc35f2 deletion (Fig. 6B) or famotidine (Fig. 6C). Instead, we discovered that losartan and naringin, which are inhibitors for the OATP family (Rebello et al., 2012; De Bruyn et al., 2013), significantly suppressed the $V_{\text {brain }}$ of YM155 (Fig. 6D). Similarly, the $V_{\text {brain }}$ of pitavastatin, the permeability of which across the BBB is determined by OATP1A4 (Ose et al., 2010), was significantly decreased by these inhibitors.

To further explore this possibility, the uptake of YM155 was determined in HEK293 cells expressing mouse OATP1A4 and human OATP1A2, the human ortholog of OATP1A4. Both types of cells displayed significant YM155 uptake (Supplemental Fig. 8, A and B). The uptake mediated by mouse OATP1A 4 was saturable, with a $K_{\mathrm{m} \text {, app }}$ value of $5.81 \pm 0.99 \mu \mathrm{M}$ (data not shown)

\section{Discussion}

In the development of drugs for treating brain diseases, their successful passage through the BBB is one of the critical factors. Therefore, understanding transport mechanisms for drugs at the BBB is of great importance. In the present study, we aimed to investigate the significance of SLC35F2 as a drug transporter within the BBB.

Immunoblotting clearly demonstrated the abundant expression of SLC35F2 in the isolated mouse and monkey BBB fraction compared with levels in the brain (Fig. 1, A and B). We also found that SLC35F2 expression was highly induced in hiPS-BMECs compared with levels in hiPS (Fig. 4A). To identify the direction of SLC35F2-mediated transport across the BBB, identifying the membrane localization of SLC35F2 is essential. We observed that hSLC35F2 was predominantly localized in the apical membrane of MDCKII cells transfected with hSLC35F2-HA (Fig. 1C), and of hiPS-BMECs (Fig. 5B), but we could not successfully detect specific SLC35F2 staining in mouse brain by immunohistochemistry (data not shown). Endothelial cells are polarized cells, and their apical membrane is exposed to the blood in vivo; therefore, it is reasonable that active efflux transporters such as P-gp and BCRP, which are located on the apical membrane, limit the distribution of their substrates from the circulation into the CNS (Demeule et al., 2002; Sasongko et al., 2005; Nicolazzo and Katneni, 2009). As SLC35F2 is also predominantly expressed in the apical membrane, it is likely to facilitate the blood-to-brain transport of its substrates. Indeed, a series of in vitro studies using famotidine or SLC35F2 knockdown or knockout in three cellular models of the primate BBB support the crucial role of SLC35F2 in YM155 uptake (Fig. 2D; Fig. 3C; Fig. 4F). Furthermore, in the BBB models that form tight monolayers, SLC35F2-mediated uptake of YM155 was remarkable from the apical side, and thereby facilitated the apical-to-basal transport across the monolayer (Figs. 3B; Fig. 5, D and $\mathrm{E})$.

We found a discrepancy in the dominant direction in the transcellular transport of YM155 between monkey BMECs and hiPS-BMECs. The B-to-A transport was greater than the A-to-B transport in monkey BMECs but was vice versa in hiPS-BMECs. YM155 is a substrate of P-gp (Iwai et al., 2011); therefore, it is reasonable to assume P-gp played a role in limiting the A-to-B transport of YM155 and in facilitating the B-to-A transport in the BBB, as observed for other P-gp substrates (Mizuno et al., 2003). Monkey BMECs obviously retain P-gp activity (Supplemental Fig. 5B), whereas hiPS-BMECs lack P-gp (Kurosawa et al., 2018). In addition, because YM155 is a water-soluble compound 
A

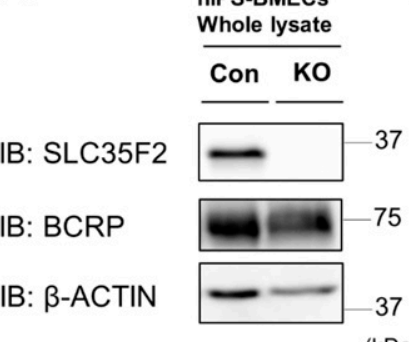

(kDa)

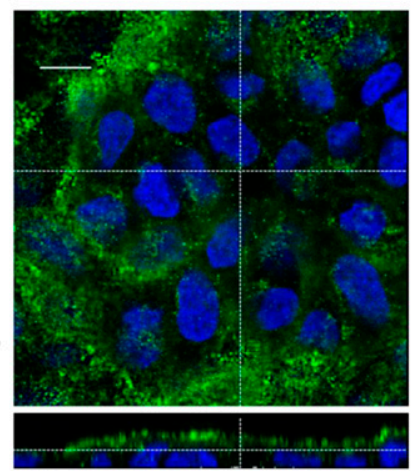

D

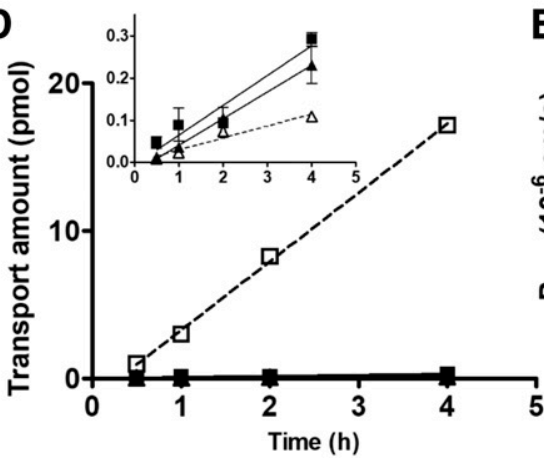

E

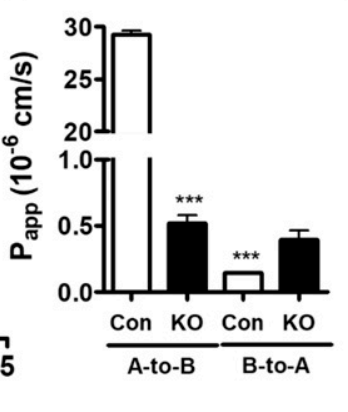

C SLC35F2 KO

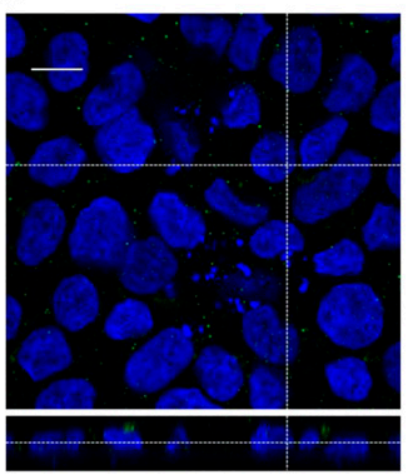

$\mathbf{F}$

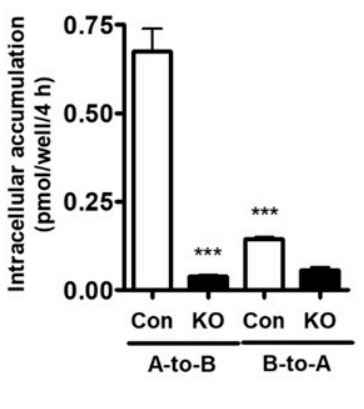

Fig. 5. Effects of SLC35F2 knockout on YM155 permeability across hiPS-BMECs. (A) Immunoblotting of whole cell lysates of hiPSBMECs confirmed SLC35F2 knockout in SLC35F2 single guide RNA-treated hiPSBMECs (KO) compared with GFP sgRNAtreated hiPS-BMECs (Con). (B and C) hSLC35F2 localization in control (A) and SLC35F2 KO (B) hiPS-BMECs detected by confocal laser scanning microscopy. Green and blue signals represent SLC35F2 and nuclear staining, respectively. The upper sections illustrate the horizontal image, and the lower sections illustrate the vertical image. Scale bars represent $10 \mu \mathrm{m}$. (D) YM155 $(0.1 \mu \mathrm{M})$ permeable amount of A-to-B transport across control hiPS-BMECs (open squares) and SLC35F2 KO hiPS-BMECs (closed squares), and B-toA transport across control hiPS-BMECs (open triangles) and SLC35F2 KO hiPS-BMECs (closed triangles), respectively. The upper figure illustrates the permeable amount except for the data of control A-to-B transport. (E and F) Calculated permeability coefficient (E) and intracellular accumulation (F) of $0.1 \mu \mathrm{M}$ YM155 in control hiPS-BMECs (open bars) and SLC35F2 KO hiPS-BMECs (closed bars). The error bars represent the S.E.M. $(n=4$, mean \pm S.E.). $* * * P<0.001$ vs. A-to-B transport in control hiPS-BMECs. The experiments were repeated at least twice. IB, immunoblotting; $\mathrm{KO}$, knockout. with poor permeability across lipid membranes without transporters (Fig. 5D), it is also possible that other transporters contribute to the transcellular transport across monolayers, such as uptake and efflux across the basolateral membrane.

Among the models tested, monkey primary BMECs appear to be the cellular model of the BBB that is most relevant to the BBB in vivo in terms of the tightness and activities of transporters. However, we observed batch differences in the quality of this model. Among those tested, lot 24E50, in which we detected the famotidine sensitivity of YM155 A-to-B transport (Fig. 3B), displayed the highest transendothelial electrical resistance (above $330 \Omega \times \mathrm{cm}^{2}$ ) and the lowest Lucifer yellow paracellular transport (25-35 pmol/4 hours; Supplemental Fig. 5A). The famotidine sensitivity of YM155 A-to-B transport paralleled the level of Lucifer yellow transport and the B-to-A/A-to-B ratio of quinidine, which could be employed as a biomarker for the tightness of endothelial cells and the activities of transporters in this system
A

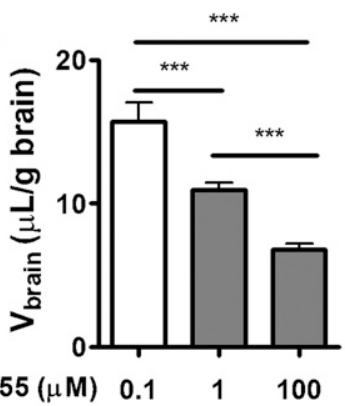

B

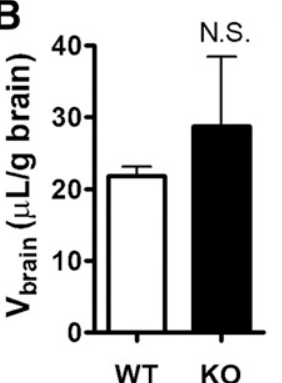

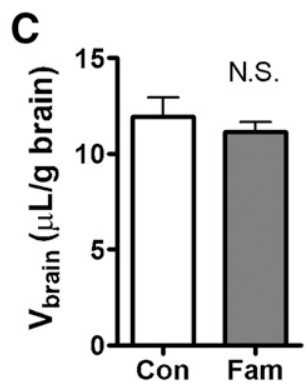

Fig. 6. Characterization of YM155 transport in the mouse BBB. (A-D) YM155 and pitavastatin brain distribution volumes were evaluated in an in situ brain perfusion test in mice. (A) Concentration dependence of YM155 brain distribution volume. (B) YM155 brain distribution volume in WT and Slc35f2 ${ }^{-/}$(KO) mice. (C) Effect of $1 \mathrm{mM}$ famotidine (Fam) on YM155 brain distribution compared with inhibitor free conditions (Con) in WT mice. (D) Effects of $100 \mu \mathrm{M}$ losartan (Los) and $100 \mu \mathrm{M}$ naringin (Nar) on YM155 and pitavastatin brain distribution compared with inhibitor free conditions (Con) in WT mice. The vertical bars represent the S.E.M. $(n=3-5$, mean \pm S.E. $)$. $* P<0.05 ; * * P<0.01 ; * * * P<0.001$ vs. control uptake. N.S indicates $P \geq 0.05$. The experiments were repeated at least twice. KO, knockout.

D

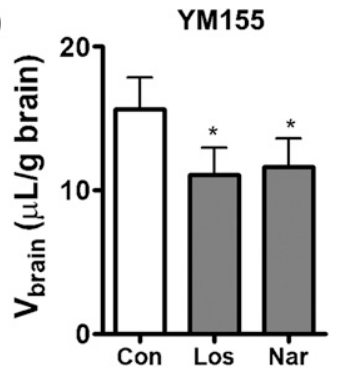

Pitavastatin

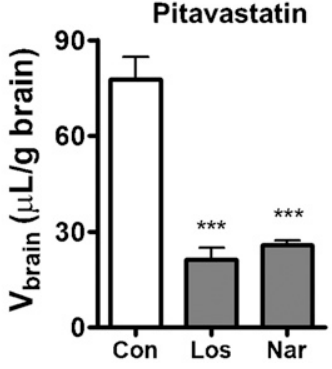


(Supplemental Table 3). There remains room for improvement of this model for use in drug transport studies.

Contrary to expectations based on in vitro data, an in situ brain perfusion test in mice elucidated a limited contribution of SLC35F2 to YM155 uptake by the brain, although YM155 is a substrate of mouse SLC35F2 (Fig. 6, B and C). Instead, YM155 transport was likely mediated by OATP1A4 (Fig. 6D; Supplemental Fig. 8B; Table 1). Previously, we reported a species difference between OATP1A4 and OATP1A2 in their specificities toward cationic substrates, triptans, and sulpiride (Liu et al., 2015; Sano et al., 2018). However, YM155 was transported by OATP1A2. It should be noted that, unlike rodent OATP1A4, OATP1A2 protein expression levels were fairly low or undetectable in the human BBB (Uchida et al., 2011)(Billington et al., 2019). As a homolog of OATP1A2, OATP2B1 protein was detectable in the human $\mathrm{BBB}$ by liquid chromatography-tandem mass spectrometry analysis (Billington et al., 2019). We could not detect any specific uptake of YM155 by this transporter (data not shown). These results suggest the possibility of species differences between rodents and primates in the transporters responsible for YM155 transport across the BBB due to the abundance of OATP1As. Direct demonstration of the significance of SLC35F2 in YM155 transport across the in vivo primate $\mathrm{BBB}$ remains a challenge. However, ${ }^{11} \mathrm{C}$-labeled YM155 was recently reported as a positron emission tomography probe for assessing in vivo tissue distribution (Murakami et al., 2013); therefore, identification of an in vivo inhibitor for SLC35F2 will be required to achieve this goal.

The present study highlighted SLC35F2 as a drug transporter in the $\mathrm{BBB}$ that can facilitate blood-to-CNS drug transport in three different cellular models of the primate BBB, although the in vivo relevance of this transporter needs to be examined in future studies. Other than YM155, we could only identify famotidine as a SLC35F2 substrate (Supplemental Fig. 4, A and B). Based on our studies, the substrate specificity appears to be narrower than the well characterized organic cation transporters such as OCT1 and OCT2. SLC35F2 displayed a similar inhibition profile to the $\mathrm{H}^{+} / \mathrm{OC}$ antiporter (Table 2) (Okura et al., 2008; Kitamura et al., 2014; Kurosawa et al., 2017); however, SLC35F2 did not facilitate the uptake of reported $\mathrm{H}^{+} / \mathrm{OC}$ antiporter substrates such as clonidine and nicotine (Supplemental Fig. 4A). Moreover, it was unlikely that YM155 transport mediated by SLC35F2 coupled with the proton gradient (Supplemental Fig. 4C). These data suggest SLC35F2 is not an entity of the $\mathrm{H}^{+} / \mathrm{OC}$ antiporter for hydrophobic organic cations. Because of the species difference in the transporter responsible for the BBB permeability of YM155, we deduced that YM155 functions as an organic anion transporter. However, none of the OAT and OATP substrates, including folic acid, estrone-3-sulfate, and hippuric acid, were transported by SLC35F2 (data not shown). A nutrition transporter, SLC19A3, was originally identified as a transporter for thiamine that accepts drugs as substrates (Liang et al., 2015). SLC35F2 may serve as a transporter for essential compounds in the CNS under normal and/or diseased conditions. Considering the future applications for CNS drug delivery, extensive studies are required to elucidate the substrate recognition profile of SLC35F2.

In conclusion, the present study demonstrated that SLC35F2 is a functional drug transporter in the BBB and that it facilitates YM155 uptake and A-to-B transport in various cellular models of the primate BBB. Our findings contribute to an improved understanding of the drug transport mechanisms in the $\mathrm{BBB}$ and to the construction of better drug development strategies for brain targeting or avoiding CNS side effects.

\section{Authorship Contributions}

Participated in research design: Mochizuki, Mizuno, Kusuhara.

Conducted experiments: Mochizuki, Kurosawa, Yamaguchi, Higuchi, Tega.
Contributed new reagents or analytic tools: Kurosawa, Yamaguchi, Higuchi,

Tega, Nozaki, Kawabata, Deguchi.

Performed data analysis: Mochizuki.

Wrote or contributed to the writing of the manuscript: Mochizuki, Mizuno, Kusuhara.

\section{References}

Arakawa R, Okumura M, Ito H, Takano A, Takahashi H, Takano H, Maeda J, Okubo Y, and Suhara T (2010) Positron emission tomography measurement of dopamine $\mathrm{D}_{2}$ receptor occupancy in the pituitary and cerebral cortex: relation to antipsychotic-induced hyperprolactinemia. J Clin Psychiatry 71:1131-1137.

Billington S, Salphati L, Hop CECA, Chu X, Evers R, Burdette D, Rowbottom C, Lai Y, Xiao G Humphreys WG, et al. (2019) Interindividual and regional variability in drug transporter abundance at the human brain barrier measured by quantitative targeted proteomics. Clinical pharmacology and therapeutics 106 (1):228-237.

Dagenais C, Rousselle C, Pollack GM, and Scherrmann JM (2000) Development of an in situ mouse brain perfusion model and its application to mdrla P-glycoprotein-deficient mice. J Cereb Blood Flow Metab 20:381-386.

Daneman R, Zhou L, Agalliu D, Cahoy JD, Kaushal A, and Barres BA (2010) The mouse bloodbrain barrier transcriptome: a new resource for understanding the development and function of brain endothelial cells. PLoS One 5:e13741.

De Bruyn T, van Westen GJP, Ijzerman AP, Stieger B, de Witte P, Augustijns PF, and Annaert PP (2013) Structure-based identification of OATP1B1/3 inhibitors. Mol Pharmacol 83:1257-1267.

Deguchi T, Kusuhara H, Takadate A, Endou H, Otagiri M, and Sugiyama Y (2004) Characterization of uremic toxin transport by organic anion transporters in the kidney. Kidney Int $\mathbf{6 5}$ : $162-174$.

Demeule M, Régina A, Jodoin J, Laplante A, Dagenais C, Berthelet F, Moghrabi A, and Béliveau $\mathrm{R}$ (2002) Drug transport to the brain: key roles for the efflux pump P-glycoprotein in the bloodbrain barrier. Vascul Pharmacol 38:339-348.

Eigenmann DE, Xue G, Kim KS, Moses AV, Hamburger M, and Oufir M (2013) Comparative study of four immortalized human brain capillary endothelial cell lines, hCMEC/D3, hBMEC TY10, and BB19, and optimization of culture conditions, for an in vitro blood-brain barrier model for drug permeability studies. Fluids Barriers CNS 10:33.

Enokizono J, Kusuhara H, Ose A, Schinkel AH, and Sugiyama Y (2008) Quantitative investigation of the role of breast cancer resistance protein (Bcrp/Abcg2) in limiting brain and testis penetration of xenobiotic compounds. Drug Metab Dispos 36:995-1002.

Hayashi H, Naoi S, Hirose Y, Matsuzaka Y, Tanikawa K, Igarashi K, Nagasaka H, Kage M, Inui A, and Kusuhara H (2016) Successful treatment with 4-phenylbutyrate in a patient with benign recurrent intrahepatic cholestasis type 2 refractory to biliary drainage and bilirubin absorption. Hepatol Res 46:192-200.

Higuchi K, Kitamura A, Okura T, and Deguchi Y (2015) Memantine transport by a proton-coupled organic cation antiporter in hCMEC/D 3 cells, an in vitro human blood-brain barrier model. Drug Metab Pharmacokinet 30:182-187.

Iwai M, Minematsu T, Li Q, Iwatsubo T, and Usui T (2011) Utility of P-glycoprotein and organic cation transporter 1 double-transfected LLC-PK1 cells for studying the interaction of YM155 monobromide, novel small-molecule survivin suppressant, with P-glycoprotein. Drug Metab Dispos 39:2314-2320.

Kitamura A, Higuchi K, Okura T, and Deguchi Y (2014) Transport characteristics of tramadol in the blood-brain barrier. J Pharm Sci 103:3335-3341.

Koepsell H, Lips K, and Volk C (2007) Polyspecific organic cation transporters: structure, function, physiological roles, and biopharmaceutical implications. Pharm Res 24:1227-1251.

Kubo Y, Seko N, Usui T, Akanuma S, and Hosoya K (2016) Lysosomal trapping is present in retinal capillary endothelial cells : insight into its influence on cationic drug transport at the inner blood - retinal barrier. Biol Pharm Bull 39:1319-1324.

Kurosawa T, Higuchi K, Okura T, Kobayashi K, Kusuhara H, and Deguchi Y (2017) Involvement of proton-coupled organic cation antiporter in varenicline transport at blood-brain barrier of rats and in human brain capillary endothelial cells. J Pharm Sci 106:2576-2582.

Kurosawa T, Tega Y, Higuchi K, Yamaguchi T, Nakakura T, Mochizuki T, Kusuhara H, Kawabata $\mathrm{K}$, and Deguchi Y (2018) Expression and functional characterization of drug transporters in brain microvascular endothelial cells derived from human induced pluripotent stem cells. Mol Pharm 15:5546-5555.

Liang X, Chien H, Yee SW, Giacomini MM, Chen EC, Piao M, Hao J, Twelves J, Twelves J, Lepist E, et al. (2015) Metformin is a substrate and inhibitor of the human thiamine transporter, THTR - 2 (SLC19A3). Molecular Pharmaceutics 12:4301-4310.

Lippmann ES, Al-Ahmad A, Azarin SM, Palecek SP, and Shusta EV (2014) A retinoic acidenhanced, multicellular human blood-brain barrier model derived from stem cell sources. Sci Rep 4:4160.

Lippmann ES, Azarin SM, Kay JE, Nessler RA, Wilson HK, Al-Ahmad A, Palecek SP, and Shusta EV (2012) Derivation of blood-brain barrier endothelial cells from human pluripotent stem cells. Nat Biotechnol 30:783-791.

Liu H, Yu N, Lu S, Ito S, Zhang X, Prasad B, He E, Lu X, Li Y, Wang F, et al. (2015) Solute carrier family of the organic anion-transporting polypeptides 1A2- MadinDarby canine kidney II: a promising in vitro system to understand the role of organic anion-transporting polypeptide $1 \mathrm{~A} 2$ in blood-brain barrier drug penetration. Drug Metab Dispos 43:1008-1018.

Minematsu T, Iwai M, Sugimoto K, Shirai N, Nakahara T, Usui T, and Kamimura H (2009) Carrier-mediated uptake of 1-(2-methoxyethyl)-2-methyl-4,9-dioxo-3-(pyrazin-2-ylmethyl)-4,9dihydro-1H-naphtho[2,3-d]imidazolium bromide (YM155 monobromide), a novel smallmolecule survivin suppressant, into human solid tumor and lymphoma cells. Drug Metab Dispos 37:619-628.

Minematsu T, Iwai M, Umehara K, Usui T, and Kamimura H (2010) Characterization of human organic cation transporter 1 (OCT1/SLC22A1)- and OCT2 (SLC22A2)-mediated transport of 1(2-methoxyethyl)-2-methyl-4,9-dioxo-3-(pyrazin-2-ylmethyl)- 4,9-dihydro-1H-naphtho[2,3-d] imidazolium bromide (YM155 monobromide), a novel small molecule survivin suppressant. Drug Metab Dispos 38:1-4.

Mizuno N, Niwa T, Yotsumoto Y, and Sugiyama Y (2003) Impact of drug transporter studies on drug discovery and development. Pharmacol Rev 55:425-461. 
Mizuno T, Hayashi H, and Kusuhara H (2015) Cellular cholesterol accumulation facilitates ubiquitination and lysosomal degradation of cell surface-resident ABCA1. Arterioscler Thromb Vasc Biol 35:1347-1356.

Murakami Y, Matsuya T, Kita A, Yamanaka K, Noda A, Mitsuoka K, Nakahara T, Miyoshi S, and Nishimura S (2013) Radiosynthesis, biodistribution and imaging of [11C]YM155, a novel survivin suppressant, in a human prostate tumor-xenograft mouse model. Nucl Med Biol 40: 221-226.

Nakagawa S, Deli MA, Nakao S, Honda M, Hayashi K, Nakaoke R, Kataoka Y, and Niwa M (2007) Pericytes from brain microvessels strengthen the barrier integrity in primary cultures of rat brain endothelial cells. Cell Mol Neurobiol 27:687-694

Nicolazzo JA and Katneni K (2009) Drug transport across the blood-brain barrier and the impact of breast cancer resistance protein (ABCG2). Curr Top Med Chem 9:130-147.

Okura T, Hattori A, Takano Y, Sato T, Hammarlund-Udenaes M, Terasaki T, and Deguchi Y (2008) Involvement of the pyrilamine transporter, a putative organic cation transporter, in bloodbrain barrier transport of oxycodone. Drug Metab Dispos 36:2005-2013.

Okura T, Kato S, and Deguchi Y (2014) Functional expression of organic cation/carnitine transporter 2 (OCTN2/SLC22A5) in human brain capillary endothelial cell line hCMEC/D3, a human blood-brain barrier model. Drug Metab Pharmacokinet 29:69-74.

Ose A, Kusuhara H, Endo C, Tohyama K, Miyajima M, Kitamura S, and Sugiyama Y (2010) Functional characterization of mouse organic anion transporting peptide 1a4 in the uptake and efflux of drugs across the blood-brain barrier. Drug Metab Dispos 38 $168-176$.

Radic-Sarikas B, Halasz M, Huber KVM, Winter GE, Tsafou KP, Papamarkou T, Brunak S, Kolch W, and Superti-Furga G (2017) Lapatinib potentiates cytotoxicity of YM155 in neuroblastoma via inhibition of the ABCB1 efflux transporter. Sci Rep 7:3091.

Rebello S, Zhao S, Hariry S, Dahlke M, Alexander N, Vapurcuyan A, Hanna I, and Jarugula V (2012) Intestinal OATP1A2 inhibition as a potential mechanism for the effect of grapefruit juice on aliskiren pharmacokinetics in healthy subjects. Eur J Clin Pharmacol 68:697-708.

Ronaldson PT, Finch JD, Demarco KM, Quigley CE, and Davis TP (2011) Inflammatory pain signals an increase in functional expression of organic anion transporting polypeptide 1a4 at the blood- brain barrier. J Pharmacol Exp Ther 336:827-839.

Sano Y, Mizuno T, Mochizuki T, Uchida Y, Umetsu M, Terasaki T, and Kusuhara H (2018) Evaluation of organic anion transporter 1A2-knock-in mice as a model of human blood-brain barrier. Drug Metab Dispos 46:1767-1775.

Sarangi A, Bupp K, and Roth MJ (2007) Identification of a retroviral receptor used by an envelope protein derived by peptide library screening. Proc Natl Acad Sci USA 104:11032-11037.

Sasongko L, Link JM, Muzi M, Mankoff DA, Yang X, Collier AC, Shoner SC, and Unadkat JD (2005) Imaging P-glycoprotein transport activity at the human blood-brain barrier with positron emission tomography. Clin Pharmacol Ther 77:503-514.
Shimomura K, Okura T, Kato S, Couraud P-O, Schermann J-M, Terasaki T, and Deguchi Y (2013) Functional expression of a proton-coupled organic cation $(\mathrm{H}+/ \mathrm{OC})$ antiporter in human brain capillary endothelial cell line hCMEC/D3, a human blood-brain barrier model. Fluids Barriers CNS 10:8.

Srinivasan B, Kolli AR, Esch MB, Abaci HE, Shuler ML, and Hickman JJ (2015) TEER measurement techniques for in vitro barrier model systems. J Lab Autom 20:107-126.

Suzuki T, Aoyama T, Suzuki N, Kobayashi M, Fukami T, Matsumoto Y, and Tomono K (2016) Involvement of a proton-coupled organic cation antiporter in the blood-brain barrier transport of amantadine. Biopharm Drug Dispos 37:323-335.

Tsuruya Y, Kato K, Sano Y, Imamura Y, Maeda K, Kumagai Y, Sugiyama Y, and Kusuhara H (2016) Investigation of endogenous compounds applicable to drug-drug interaction studies involving the renal organic anion transporters, OAT1 and OAT3, in humans. Drug Metab Dispos 44:1925-1933.

Uchida Y, Ohtsuki S, Katsukura Y, Ikeda C, Suzuki T, Kamiie J, and Terasaki T (2011) Quantitative targeted absolute proteomics of human blood-brain barrier transporters and receptors. Journal of Neurochemistry 117 (2):333-345.

Weksler B, Romero IA, and Couraud P-O (2013) The hCMEC/D3 cell line as a model of the human blood brain barrier. Fluids Barriers CNS 10:16.

Weksler BB, Subileau EA, Perrière N, Charneau P, Holloway K, Leveque M, Tricoire-Leignel H, Nicotra A, Bourdoulous S, Turowski P, et al. (2005) Blood-brain barrier-specific properties of a human adult brain endothelial cell line. FASEB $J$ 19:1872-1874.

Winter GE, Radic B, Mayor-Ruiz C, Blomen VA, Trefzer C, Kandasamy RK, Huber KVM, Gridling M, Chen D, Klampfl T, et al. (2014) The solute carrier SLC35F2 enables YM155mediated DNA damage toxicity. Nat Chem Biol 10:768-773.

Yamaoka K, Tanigawara Y, Nakagawa T, and Uno T (1981) A pharmacokinetic analysis program (multi) for microcomputer. J Pharmacobiodyn 4:879-885.

Yang S, Jin H, and Zhao Z (2018) Paracellular tightness and the functional expression of efflux transporters P-gp and BCRP in bEnd3 cells. Neurol Res 40:644-649.

Zhang Y, Chen K, Sloan SA, Bennett ML, Scholze AR, O'Keeffe S, Phatnani HP, Guarnieri P, Caneda C, Ruderisch N, et al. (2014) An RNA-sequencing transcriptome and splicing database of glia, neurons, and vascular cells of the cerebral cortex. J Neurosci 34:11929-11947.

Address correspondence to: Dr. Hiroyuki Kusuhara, Laboratory of Molecular Pharmacokinetics, Graduate School of Pharmaceutical Sciences, The University of Tokyo, 7-3-1 Hongo, Bunkyo-ku, Tokyo 113-0033, Japan. E-mail: kusuhara@mol.f. u-tokyo.ac.jp 